OPEN ACCESS

Edited by:

Eugen Tausch,

University of UIm, Germany

Reviewed by:

Lucrecia Yañez San Segundo,

Marqués de Valdecilla University

Hospital, Spain

Nicoletta Coccaro,

University of Bari Aldo Moro, Italy

*Correspondence:

Karla Plevova

karla.plevova@mail.muni.cz

Specialty section:

This article was submitted to Hematologic Malignancies,

a section of the journal

Frontiers in Oncology

Received: 06 September 2021 Accepted: 01 November 2021 Published: 26 November 2021

Citation:

Zavacka K and Plevova K (2021)

Chromothripsis in Chronic

Lymphocytic Leukemia: A Driving

Force of Genome Instability.

Front. Oncol. 11:771664.

doi: 10.3389/fonc.2021.771664

\section{Chromothripsis in Chronic Lymphocytic Leukemia: A Driving Force of Genome Instability}

\author{
Kristyna Zavacka ${ }^{1,2}$ and Karla Plevova ${ }^{1,2,3 *}$ \\ 1 Department of Internal Medicine - Hematology and Oncology, University Hospital Brno \& Faculty of Medicine, Masaryk \\ University, Brno, Czechia, ${ }^{2}$ Center of Molecular Medicine, Central European Institute of Technology, Masaryk University, \\ Brno, Czechia, ${ }^{3}$ Institute of Medical Genetics and Genomics, University Hospital Brno \& Masaryk University, Brno, Czechia
}

Chromothripsis represents a mechanism of massive chromosome shattering and reassembly leading to the formation of derivative chromosomes with abnormal functions and expression. It has been observed in many cancer types, importantly, including chronic lymphocytic leukemia (CLL). Due to the associated chromosomal rearrangements, it has a significant impact on the pathophysiology of the disease. Recent studies have suggested that chromothripsis may be more common than initially inferred, especially in CLL cases with adverse clinical outcome. Here, we review the main features of chromothripsis, the challenges of its assessment, and the potential benefit of its detection. We summarize recent findings of chromothripsis occurrence across hematological malignancies and address its causes and consequences in the context of CLL clinical features, as well as chromothripsis-related molecular abnormalities described in published CLL studies. Furthermore, we discuss the use of the current knowledge about genome functions associated with chromothripsis in the optimization of treatment strategies in CLL.

Keywords: chromothripsis, chronic lymphocytic leukemia, complex chromosomal rearrangements, copy number alterations, genomic array, paired-end sequencing, oncogene amplification, tumor suppressor inactivation

\section{INTRODUCTION}

Chronic lymphocytic leukemia (CLL) is the most common adult leukemia in Western countries with a highly variable clinical course. Several recurrent chromosomal alterations have been associated with prognosis and may guide risk-adapted therapy. Besides deletions on chromosomes 11,13,17, and trisomy 12, high genomic complexity (high-GC) has also been recognized as a feature with prognostic value $(1,2)$ and is associated with poor clinical outcome $(3,4)$. Cytogenetics and array-based methods define high-GC as five or more chromosomal defects $(1,2)$. In many instances, highly complex karyotypes can be caused by chromothripsis (cth) (5), a genomic event by which a single or a limited number of chromosomes are shattered into pieces, followed by error-prone reassembly (6-9).

Among all cancers, it was CLL where the evidence of cth was reported for the first time. This finding was made already a decade ago via the whole-genome sequencing screening of $10 \mathrm{CLL}$ 
patients (5). In a sample from a 62-year-old woman without any previous CLL treatment, a massive rearrangement of chromosomal arm 4q and focal alterations on chromosomes 1, 12 , and 15 were found, showing striking patterns. It was proved that this complex genomic remodeling had occurred before the diagnosis and persisted until the rapid disease relapse after alemtuzumab treatment without further evolution. The phenomenon was termed chromothripsis (from Greek; chromos for chromosome, thripsis for shattering into pieces) and was subsequently observed in many other tumor types (5, 10-15).

In contrast to the traditional view of tumorigenesis as the multi-step accumulation of mutations, cth arises via a single devastating event. Within a single cell division, tens to hundreds of DNA double-strand breaks are generated and imperfectly assembled into derivative chromosomes, most often via nonhomologous end joining (NHEJ), whereas some fragments can be lost (Figure 1A). The massively rearranged genomes of the cells that survive such an event propagate in daughter clones and are likely to have gained a strong selection advantage, as cth could disrupt the functions of tumor suppressors, support the oncogene amplification, and/or give rise to pathogenic gene fusions. Thus, cth is a potential driving force of malignant transformation and tumor progression.

\section{DETECTION OF CHROMOTHRIPSIS- LIKE PATTERNS}

Cth is characterized by several hallmarks that set it apart from other complex genomic changes: (a) occurrence of tens to hundreds of chromosomal rearrangements with pronounced clustering, (b) random orientation of rearrangements resulting in equal representation of deletions, inversions, and tandem duplications, (c) copy-number alterations (CNAs) oscillating between two (occasionally three) copy-number states, (d) alterations of segments that retained heterozygosity and segments with loss-of-heterozygosity (LOH), (e) structural rearrangements displaying a bias toward occurring on a single chromosome homolog, and (f) presence of double-minute chromosomes $(5,15)$. The evidence of different cth patterns in various cancer types and among individual cases (21) suggests different mechanisms of its origin. The mechanisms, presumed most frequently to cause cth, include asynchronous DNA replication in abnormal nuclear structures called micronuclei $(6,22,22)$ (Figure 1B) and the fragmentation of dicentric chromosomes resulting from the telomere crisis due to their extreme shortening (23) (Figure 1C).

Since the genomic profile originating in cth could be similar to stepwise processes, the detection of cth is often challenging. Therefore, a set of criteria was generated for accurate and reproducible cth inference (7). Most of these criteria take into account the entire set of structural rearrangements that occurred on a chromosome, including the relative order and orientation of rearranged segments. They are typically detected using wholegenome paired-end DNA sequencing. Copy-number states can also be analyzed by array-based comparative genomic hybridization $(\mathrm{aCGH})$ or single nucleotide polymorphism (SNP) arrays. However, for the most accurate detection of cth, it is desirable to use a complex approach that combines sequencing genomic methods with molecular cytogenetics and other complementary methods (24). A conventional karyotyping of metaphases can be useful to identify numerical and structural chromosomal abnormalities. Various fluorescence in situ hybridization (FISH) techniques may aid the identification of interacting chromosome partners and localization of breakpoints. Spectral karyotyping in combination with fluorescent locus-specific probes can effectively detect the double-minute chromosomes (5). Above that, RNA-Seq can assist in revealing abnormalities at the transcriptional level such as de novo fusion transcripts or abnormal gene expression, both of which can be revealed with advanced analytical methods (25).

\section{CHROMOTHRIPSIS IN HEMATOLOGICAL MALIGNANCIES}

Cth has been observed in primary tumors of various histological types, including hematological malignancies, such as lymphomas $(19,21,26)$, multiple myeloma $(11,26,27)$, myelodysplastic syndrome $(26,28,29)$, and leukemias $(2,3,5,15-21,24,30-39)$. The prevalence of cth across cancer types ranges from units to tens of percent with the highest proportions in sarcomas - up to $100 \%(5,21,40)$. However, the comparison of published studies provides only rough estimation due to different methodologies and definitions used for cth scoring. The cth frequencies observed in hematological malignancies are summarized in Table 1.

For most hematological diseases, cth provides independent prognostic information and is associated with adverse clinical outcome. In myelodysplastic syndrome, the complex chromosomal rearrangements caused by cth are related to advanced disease stages prone to transform to acute myeloid leukemia (AML); as a consequence, they recurrently involve $5 \mathrm{q}$ deletions (28). Similarly, AML patients with cth have a high recurrence of $5 \mathrm{q}$ losses, and also TP53 dysregulation and the presence of marker chromosomes (30-32). Besides that, cth appears to be mutually exclusive with FLT3 and NPM1 mutations $(30,32)$. In acute lymphoblastic leukemia (ALL), cth occurs predominantly in specific subgroups, such as early $\mathrm{T}$ cell precursor ALL $(35,37)$, iAMP21 B-ALL (36), and ataxiatelangiectasia-related T-ALL (37).

The evidence of cth cases described in CLL indicates that this phenomenon is a recurrent event. By exploring larger cohorts of CLL patients, cth was observed with frequencies from 1.2 to $10 \%$ $(2,3,5,18,21,24,38,39)$. Although the reported prevalence is relatively small, the analysis of cth-like patterns may be beneficial for clinical decision-making and precision medicine, as cth represents a driving force of genome evolution in $\operatorname{CLL}(5,16$, $17,20)$. 
A

Orighal chromosome structure

A B C D E F G H I*JKL M N O P Q R

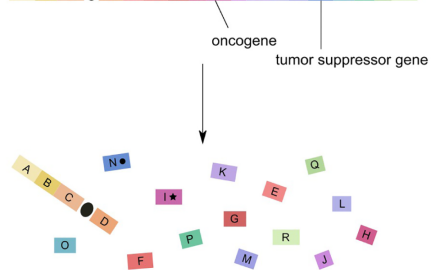

chromosome shattering

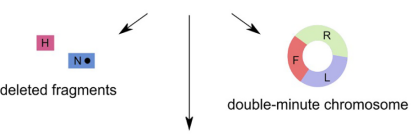

Derivative chromosome

A B C D P Q I* $1 * \exists O K$ K P M O

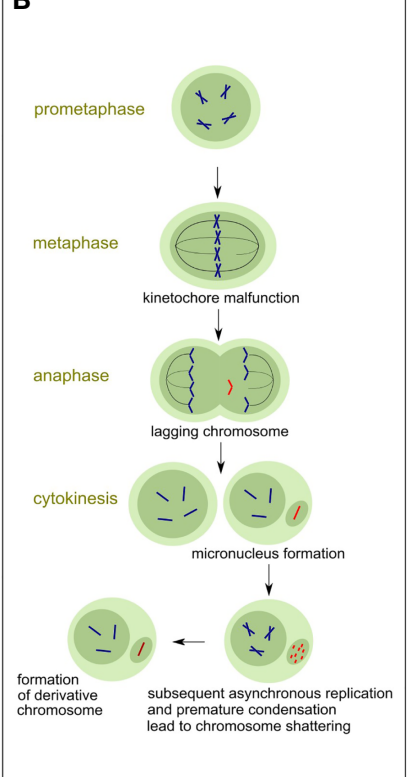

C

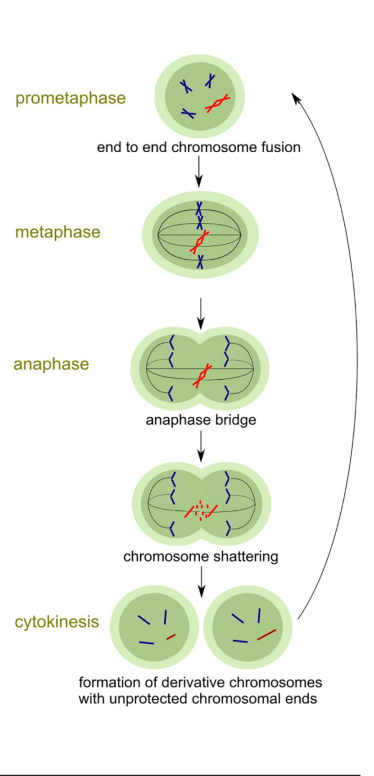

D
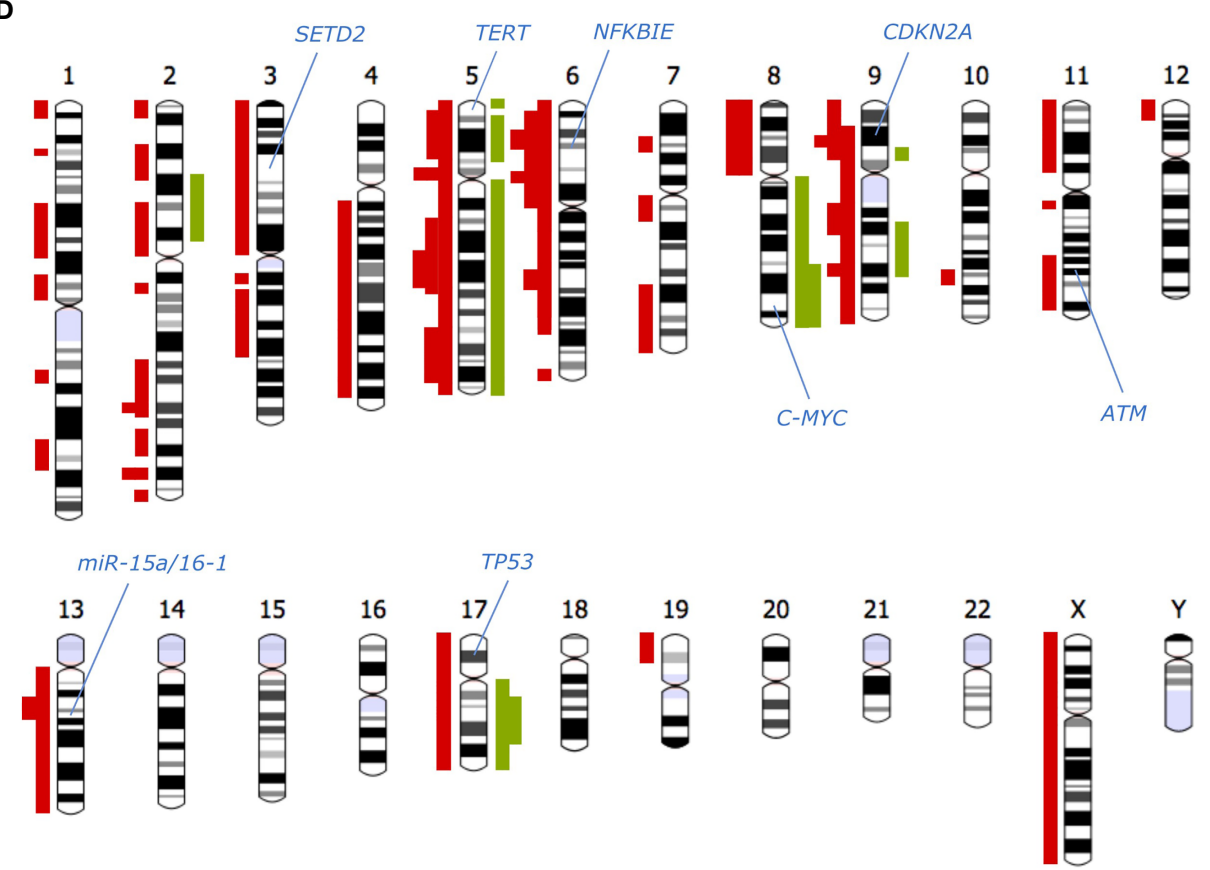

FIGURE 1 | Causes and consequences of chromothripsis. (A) Schematic model of chromosome shattering and reassembly via cth: After chromosome fragmentation, some regions are incorporated (possibly in multiple copies) into a derivative chromosome, whereas other regions can be lost or fused to episomal structures called double-minute chromosomes. (B) The micronuclei hypothesis of the cth origin: Chromosomes that are missegregated during cell division are entrapped in the micronucleus, followed by asynchronous replication compared to the main nucleus. This leads to premature chromosome condensation and shattering. Rejoining of fragments gives rise to the derivative chromosome which can subsequently be reincorporated into the main nucleus. (C) The origin of cth due to breakage-fusionbridge (BFB) cycles and telomere crisis: Chromosome ends that become unprotected due to telomere shortening are fused into a dicentric chromosome containing two centromeres. In the subsequent cell cycle, this unstable structure is pulled to opposite spindle poles forming an anaphase bridge between the two daughter cells. The rupturing bridge generates two new unprotected chromosomal ends and initiates a new round of the BFB cycle. This repeats until the derivative chromosome becomes stable. (D) Chromosomal ideograms with cth-derived gains (green) and losses (red) observed in the following CLL studies: Stephens et al., 2011 (5); Edelmann et al., 2012 (3); Pei et al., 2012 (16), Bassaganyas et al., 2013 (17); Salaverria et al., 2013 (18); Tan et al., 2015 (19); Parker et al., 2016 (20); Leeksma et al., 2021 (2). The thickness of the highlighted loci corresponds to the number of studies referring to the respective regions affected by cth. Only studies mentioning specific affected areas and distinguishing individual patients were compiled. 
TABLE 1 | Prevalence of chromothripsis in CLL and other hematological malignancies.

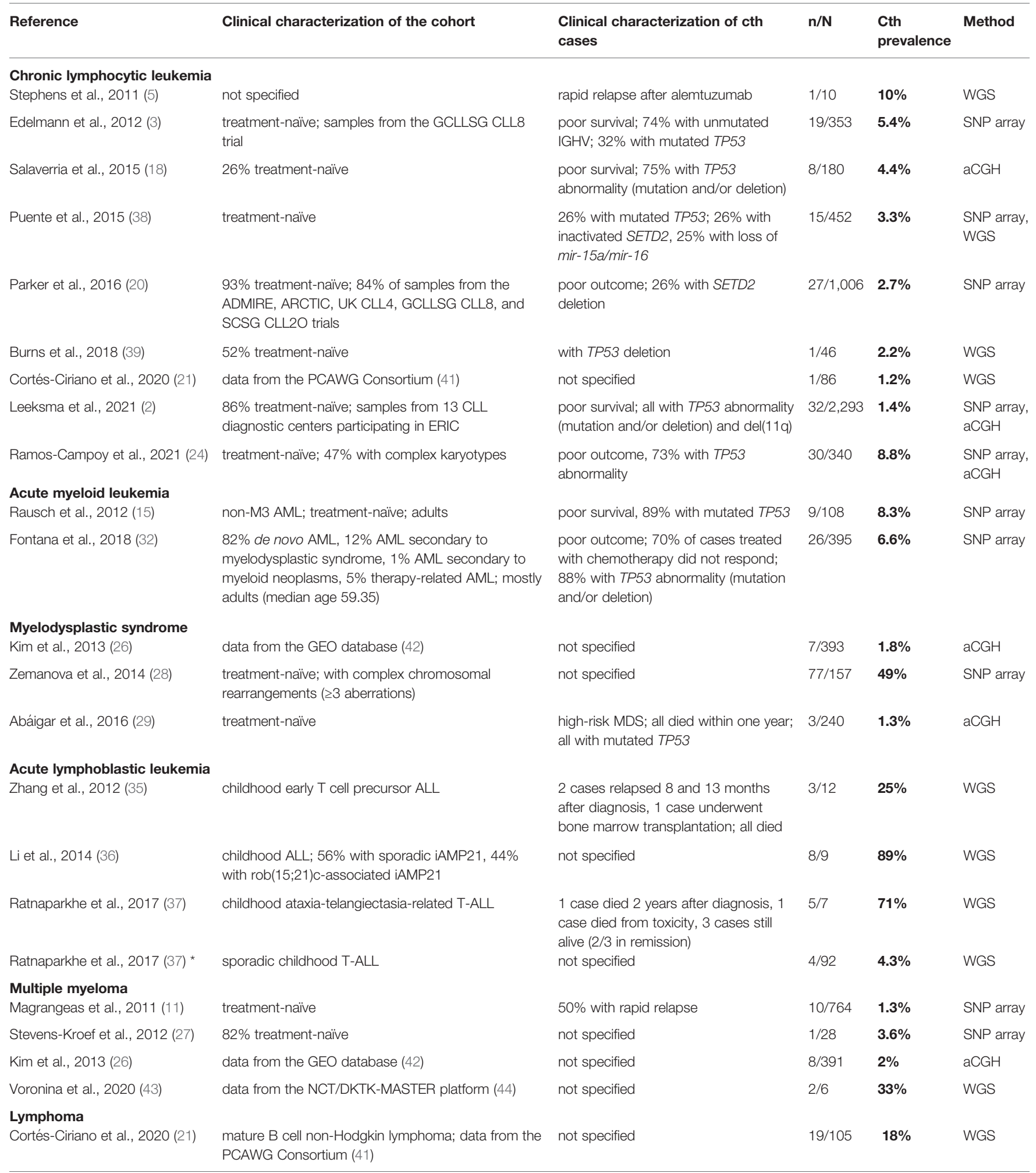

*refers to unpublished data discussed with Meijerink et al., partly published in Li et al., 2016 (45).

$n$, the number of cth cases; N, the total number of cases analyzed in the respective study; GCLLSG, German CLL Study Group; PCAWG, Pan-Cancer Analysis of Whole Genomes; ERIC, European Research Initiative on CLL; GEO, Gene Expression Omnibus; NCT/DKTK-MASTER, National Center for Tumor Diseases/German Cancer Consortium-Molecularly Aided Stratification for Tumor Eradication; WGS, whole-genome sequencing; SNP array, single-nucleotide polymorphism array; aCGH, array comparative genomic hybridization. 


\section{IMPACT OF CHROMOTHRIPSIS ON CLL ONSET AND PROGRESSION}

CLL patients with cth (cth-CLL) were shown to have inferior time to first treatment (24), progression-free survival (3), and overall survival $(2,3,18,38)$. The overall survival of cth-CLL cases was even worse than of cases with TP53 abnormality or del(11q) without cth (2). The majority of cth-CLL cases have unmutated IGHV $(3,17,24,38,39)$. Two studies concluded that cth is more frequent in the IGHV-unmutated group with statistical significance $(3,38)$. There is also a strong link between the presence of cth and high-risk genomic aberrations like del(11q) and del(17p) $(2,3,16,17,19,20,24,39)$.

Some studies reported that cth occurs before the CLL diagnosis indicating that the complex genomic remodeling could be a CLL-initiating event $(5,16)$ or one of the earliest events in the CLL pathogenesis (20). On the contrary, a case study from 2013 showed that cth is not necessarily triggering the CLL onset. In this case, cth was a consequence of previous alterations accumulated since the time of diagnosis and contributed to the increase of CLL aggressiveness, as a subclone carrying complex structural variants expanded and outbalanced the predominant tumor population before the first treatment (17). Interestingly, the cth-subclone was eradicated by chemotherapy and did not reappear throughout a 10-year follow-up period. This observation contrasts other data strongly associating cth-clones with chemotherapy resistance and/or poor clinical outcome $(2,3,5)$. That points to the substantial need for larger cohorts of cth cases to be analyzed to better understand the dynamics of cth in CLL.

\section{GENOMIC REGIONS ASSOCIATED WITH CHROMOTHRIPSIS IN CLL}

Chromosomes 2, 3, 6, 8, 9, 11, 13, and 17 were impacted by cth in CLL most frequently $(2,3,16,17,19,20,39)$ (Figure 1D). Many cth-CLL cases harbor del(17p) $(2,3,16,19,20,24,39)$ spanning the TP53 gene, the most important predictor of disease and treatment outcome (46-51). Alterations in TP53 are the most common changes associated with cth in medulloblastoma (15), acute myeloid leukemia (15), pediatric cancers (52), and CLL $(2,3,20,24,38)$. TP53 is responsible for cell cycle control, genome maintenance, and apoptosis $(53,54)$, confirming its plausible involvement in genome instability preceding cth. The frequent co-occurrence of TP53 alterations and cth in CLL supports both possibilities of their relation, i.e. cth resulting from TP53 disruption as well as cth leading to TP53 abnormalities and therefore more aggressive disease. Alterations in ATM including del(11q) and gene mutations can also explain the rise of cth considering its role in the regulation of the DNA damage response (DDR) and were observed in patients with cth $(2,3,20,39)$. In this context, Bassaganyas et al. (17) observed the $A T M^{R 189 T}$ mutation in the CLL patient two years before cth detection.
Moreover, SETD2 deletions have been associated with the loss of TP53, genomic complexity, and cth and define a subgroup of patients with poor outcome (20). The published data highlight SETD2 aberrations as a recurrent, clonal, early lossof-function event in CLL pathobiology that appears to be the result of cth and linked to aggressive disease. In this comprehensive study, 26\% CLL cases with SETD2 deletions showed evidence of cth on chromosome 3, constituting predominantly cases with ultra-high-risk CLL. Another study also proved that SETD2 inactivation is more frequent in CLL cases with cth than in non-cth cases (26\% versus $1.4 \%$ ) (38).

In the case study by Bassaganyas et al. (17), the authors found cth-derived deletion of 6q21 spanning the NFKBIE gene. In general, del $(6 \mathrm{q})$ is known to be present in $6 \%$ of CLL and linked to shorter progression-free survival $(3,46,55)$. In the reported case, the concurrent $N F K B I E^{E 285 X}$ mutation on the other allele led to the absence of a functional NFKBIE in cth-subclone. Moreover, del(10q24) involving NFKB2, a subunit of NF$\kappa \mathrm{B}$ transcription factor complex regulating the NFKBIE transcription, was observed (17).

Although seen with low frequency, there were observations of the cth-related gain of 8q (the C-MYC gene) (19), loss of chromosome 13 (mir-15a/mir-16) $(5,38)$, and loss of $14 \mathrm{q}(16)$, which are recurrently detected in CLL. Loss of 8 p, associated with a higher number of CNAs in CLL $(56,57)$, was also observed in cth-CLL (39). In addition, RNA-Seq revealed a fusion transcript of UBR2-SPATS1 in one case (17) potentially contributing to disease aggressiveness, as the UBR2 gene is involved in the cell growth controlling (58) and could have been deregulated or have gained a new function due to premature truncation and fusion with the second partner.

\section{ASSOCIATIONS OF TELOMERE BIOLOGY AND CHROMOTHRIPSIS IN CLL}

Telomere dysfunction is known to have a dynamic role in shaping a disease course in CLL $(59,60)$. Physiological telomere shortening corresponding to the number of divisions a cell goes through leads to gradual uncapping of the chromosome ends. At a certain critical point, telomeres are recognized as DNA double-strand breaks and trigger the DDR. As a consequence, the senescence and/or apoptosis checkpoints are activated to prevent neoplastic transformation (61). If protective mechanisms are compromised, cells may continue to proliferate, which results in genomic instability (62). Studies have shown that CLL cells have a close inverse correlation between telomere length and telomerase activity compared to healthy cells (63-66). This could be explained by the theory that the genomic instability associated with shorter telomeres promotes the selection of fit CLL clones that overcome senescence and sustain cell survival due to the maintenance of minimal telomere length by telomerase. It was shown that the tumor microenvironment-mediated signaling, such as BCR or PI3K signaling, contributes to telomerase activation (67). 
The dysfunctional telomeres often induce intra- or interchromosomal end fusions that can occur as clonal events. Their frequency was found to increase with the advancing disease stage in CLL (68). Such telomere fusions result in the formation of dicentric chromosomes that undergo breakage at the anaphase. This phenomenon is known as the breakagefusion-bridge (BFB) cycle (69) and can be a precursor to genomic complexity such as cth $(23,70)$ (Figure 1C). Studies described the association of short telomeres with complex karyotypes $(64,71,72)$ or with a higher number of CNAs $(73,74)$ in CLL. Unlike other tumor entities (e.g. central nervous system tumors), CLL cells were shown to have shorter telomeres in the cases with cth as compared to the cases without cth (70).

In general, the telomere length has been proposed to be an independent prognostic factor in CLL, with short telomeres being associated with adverse outcome (63-66, 73-76), the presence of $\operatorname{del}(11 q)$ and $\operatorname{del}(17 p)(64,72-75,77)$, as well as mutations in ATM and TP53 (72-74, 76-78) both of which serve as critical checkpoint genes activated upon telomere shortening. However, the association between telomere dysfunction and cth was confirmed to be independent of the TP53 mutation status in CLL (70). It has been supposed that in cases where no somatic TP53 mutation was detected, other aberrations affecting the DDR and/or potentially inducing p53 dysfunction likely allow the cell to avoid apoptosis despite telomere dysfunction. On the other hand, del(17p) treatment-naive CLL patients with cth have significantly shorter telomeres compared to those without cth (79). Moreover, loss of SMC5, which is involved in maintaining genomic stability and plays a role in telomere-related functions, might favor cth, especially when cooccurring with short telomeres and TP53 defects (79). In addition, certain CLL cases with cth-like patterns in the $5 p$ region were discovered, including gains of TERT, which encodes the telomerase reverse transcriptase (18).

It is presumed that the derivative chromosomes resulting from cth are likely stabilized hindering further progressive chromosomal cataclysm that would be incompatible with cell survival. From longitudinal observations, the chromothriptic patterns in CLL patients are either stable, in which case the relapse specimens show similar aberrations to the primary samples $(5,70)$, or they are lost by clonal selection in the relapse (17). Thus, telomere stabilization mechanisms are likely activated after the occurrence of cth to prevent continuing (and presumably lethal) genome-wide disruption.

All the mentioned findings confirm that the telomere attrition followed by end-to-end chromosome fusion and subsequent breakage leads to cth in CLL. This is followed by the establishment of telomere maintenance mechanisms that "lock-in" these alterations and prevent further lethal events. It, therefore, highlights the importance of detecting cth in the context of telomere length for risk stratification as well as for monitoring and early identification of clonal changes. Similarly, telomere maintenance mechanisms may represent a target for therapeutic intervention in cthpositive cases.

\section{CHROMOTHRIPSIS IN CLL DIAGNOSTICS AND TREATMENT}

The available data suggest the potential of cth detection for better stratification of CLL patients by recognizing cases with highly complex karyotypes and thus adverse prognosis. Studies showed that cth-CLL patients show adverse clinical course and demand an early therapeutic intervention $(2,3,5,18,19)$, often even evolving refractory disease $(3,5,20)$.

As follows from the information above, cth is a consequence of genomic instability and is associated with aberrations in specific molecular pathways $(15,22,80)$. In these cases (presumably more than in others) the cell signaling inhibitors should provide a promising outcome, similarly to the cases with defective TP53. However, no studies are available yet.

In general, the detection of cth-associated abnormalities could serve for the identification of molecular therapeutic targets. For instance, targeting oncogenes amplified via cth might provide a therapeutic benefit. Additionally, leukemic cells with cth could successfully respond to immune checkpoint blockade due to potential neoantigens generated from genomic rearrangements (81). The neoantigens were proven to bind patient-specific major histocompatibility complex molecules and to expand tumorinfiltrating $\mathrm{T}$ cell clones (82). These findings might be exploited for the development of novel immunotherapeutic approaches as well as the selection of patients to be administered immunotherapies. This strategy has already been suggested for a subset of AML patients with a high burden of alterations (32). Similarly, cth-derived fusion genes can help to increase the sensitivity of cancer cells to certain types of agents. An example is a patient with myelodysplastic syndrome, where several cryptic fusions, including ETV6-PDGFRB, were found (83). This is underlain by the fact that the myeloid neoplasms associated with $P D G F R B$ rearrangement represent a specific entity sensitive to tyrosine kinase inhibitors (84).

Besides that, a synthetic lethality approach (85) is also an option for cth-CLL treatment. This approach is based on targeting a synthetic-lethal partner of a gene that is already mutated or overexpressed - that means targeting a gene that is compensating for the loss of activity of the dysfunctional one. Simultaneous inactivation of such gene pair results in cell death (85). As the defects in the DDR mechanism are frequently associated with cth, the cells have an increased level of DNA damage and evolve new mechanisms to resist endogenous and exogenous stress. The strategy of synthetic lethality in such cases could combine current treatment modalities with drugs targeting residual DNA repair pathways that such cells are dependent on (86).

\section{CONCLUSIONS}

Based on the available data, cth is a recurrent event in CLL and could have a strong prognostic value. Although there is rapid progress in understanding molecular processes behind cth, current studies have important limitations. The biggest 
drawback is a relatively small number of CLL patients that have been analyzed so far which hampers the reproducibility of published results. Another issue is missing longitudinal observations. Most studies focus on a single time point of the disease, usually treatment-naïve. However, the information about the dynamics of the cth and the changes accompanying this event is lacking. It would be of interest to elucidate which changes precede the development of cth and which, in contrast, are more frequently its consequence. These findings would facilitate a better understanding of CLL clonal evolution and its driving forces and could reveal recurrently altered molecular pathways with different prognostic impacts.

The genomic landscape induced by cth is complex and linking cth to specific clinical outcomes is not always straightforward. The genes and genomic regions affected by cth appear to be the most important factors for the disease phenotype, not the occurrence of cth itself. This highlights the growing need for personalized medicine to be implemented into CLL treatment. Analyzing tumor samples at different time points should also be a part of the clinical program to elucidate clonal genotypes that could be therapy-resistant, which might help in therapeutic decisions along the disease course.

\section{REFERENCES}

1. Baliakas P, Jeromin S, Iskas M, Puiggros A, Plevova K, Nguyen-Khac F, et al. Cytogenetic Complexity in Chronic Lymphocytic Leukemia: Definitions, Associations, and Clinical Impact. Blood (2019) 133:1205-16. doi: 10.1182/ blood-2018-09-873083

2. Leeksma AC, Baliakas P, Moysiadis T, Puiggros A, Plevova K, van der KevieKersemaekers A-M, et al. Genomic Arrays Identify High-Risk Chronic Lymphocytic Leukemia With Genomic Complexity: A Multi-Center Study. Haematologica (2021) 106:87-97. doi: 10.3324/haematol.2019.239947

3. Edelmann J, Holzmann K, Miller F, Winkler D, Bühler A, Zenz T, et al. HighResolution Genomic Profiling of Chronic Lymphocytic Leukemia Reveals New Recurrent Genomic Alterations. Blood (2012) 120:4783-94. doi: 10.1182/blood-2012-04-423517

4. Malek SN. The Biology and Clinical Significance of Acquired Genomic Copy Number Aberrations and Recurrent Gene Mutations in Chronic Lymphocytic Leukemia. Oncogene (2013) 32:2805-17. doi: 10.1038/onc.2012.411

5. Stephens PJ, Greenman CD, Fu B, Yang F, Bignell GR, Mudie LJ, et al. Massive Genomic Rearrangement Acquired in a Single Catastrophic Event During Cancer Development. Cell (2011) 144:27-40. doi: 10.1016/ j.cell.2010.11.055

6. Zhang C-Z, Leibowitz ML, Pellman D. Chromothripsis and Beyond: Rapid Genome Evolution From Complex Chromosomal Rearrangements. Genes Dev (2013) 27:2513-30. doi: 10.1101/gad.229559.113

7. Korbel JO, Campbell PJ. Criteria for Inference of Chromothripsis in Cancer Genomes. Cell (2013) 152:1226-36. doi: 10.1016/j.cell.2013.02.023

8. Leibowitz ML, Zhang C-Z, Pellman D. Chromothripsis: A New Mechanism for Rapid Karyotype Evolution. Annu Rev Genet (2015) 49:183-211. doi: 10.1146/annurev-genet-120213-092228

9. Umbreit NT, Zhang C-Z, Lynch LD, Blaine LJ, Cheng AM, Tourdot R, et al. Mechanisms Generating Cancer Genome Complexity From a Single Cell Division Error. Science (2020) 368:eaba0712. doi: 10.1126/science.aba0712

10. Kloosterman WP, Hoogstraat M, Paling O, Tavakoli-Yaraki M, Renkens I, Vermaat JS, et al. Chromothripsis Is a Common Mechanism Driving Genomic Rearrangements in Primary and Metastatic Colorectal Cancer. Genome Biol (2011) 12:R103. doi: 10.1186/gb-2011-12-10-r103

11. Magrangeas F, Avet-Loiseau H, Munshi NC, Minvielle S. Chromothripsis Identifies a Rare and Aggressive Entity Among Newly Diagnosed Multiple Myeloma Patients. Blood (2011) 118:675-8. doi: 10.1182/blood-2011-03-344069

\section{AUTHOR CONTRIBUTIONS}

$\mathrm{KZ}$ drafted the manuscript and created figures. KP proposed the structure and supervised manuscript preparation, both authors performed the literature search and contributed to manuscript writing. Both authors contributed to the article and approved the submitted version.

\section{FUNDING}

The authors acknowledge the support by the AZV project NU2108-00237 and the program for the conceptual development of research organization FNBr 65269705 provided by the Ministry of Health of the Czech Republic, the student projects MUNI/A/ 1595/2020 and MUNI/IGA/1640/2020 provided by the Ministry of Education, Youth and Sports of the Czech Republic, and the European Regional Development Fund Project "A-C-G-T" No. CZ.02.1.01/0.0/0.0/16_026/0008448. KZ is a holder of Brno Ph.D. Talent 2019 Scholarship funded by the Brno City Municipality. The content of this manuscript is a part of the doctoral thesis of KZ.

12. Northcott PA, Shih DJH, Peacock J, Garzia L, Morrissy AS, Zichner T, et al. Subgroup-Specific Structural Variation Across 1,000 Medulloblastoma Genomes. Nature (2012) 488:49-56. doi: 10.1038/nature11327

13. Hirsch D, Kemmerling R, Davis S, Camps J, Meltzer PS, Ried T, et al. Chromothripsis and Focal Copy Number Alterations Determine Poor Outcome in Malignant Melanoma. Cancer Res (2013) 73:1454-60. doi: 10.1158/0008-5472.CAN-12-0928

14. Molenaar JJ, Koster J, Zwijnenburg DA, van Sluis P, Valentijn LJ, van der Ploeg I, et al. Sequencing of Neuroblastoma Identifies Chromothripsis and Defects in Neuritogenesis Genes. Nature (2012) 483:589-93. doi: 10.1038/ nature 10910

15. Rausch T, Jones DTW, Zapatka M, Stütz AM, Zichner T, Weischenfeldt J, et al. Genome Sequencing of Pediatric Medulloblastoma Links Catastrophic DNA Rearrangements With TP53 Mutations. Cell (2012) 148:59-71. doi: $10.1016 /$ j.cell.2011.12.013

16. Pei J, Jhanwar SC, Testa JR. Chromothripsis in a Case of TP53-Deficient Chronic Lymphocytic Leukemia. Leuk Res Rep (2012) 1:4-6. doi: 10.1016/ j.lrr.2012.09.001

17. Bassaganyas L, Beà S, Escaramís G, Tornador C, Salaverria I, Zapata L, et al. Sporadic and Reversible Chromothripsis in Chronic Lymphocytic Leukemia Revealed by Longitudinal Genomic Analysis. Leukemia (2013) 27:2376-9. doi: $10.1038 /$ leu.2013.127

18. Salaverria I, Martín-Garcia D, López C, Clot G, García-Aragonés M, Navarro A, et al. Detection of Chromothripsis-Like Patterns With a Custom Array Platform for Chronic Lymphocytic Leukemia. Genes Chromosomes Cancer (2015) 54:668-80. doi: 10.1002/gcc.22277

19. Tan L, Xu L-H, Liu H-B, Yang S-J. Small Lymphocytic Lymphoma/Chronic Lymphocytic Leukemia With Chromothripsis in an Old Woman. Chin Med J (Engl) (2015) 128:985-7. doi: 10.4103/0366-6999.154329

20. Parker H, Rose-Zerilli MJJ, Larrayoz M, Clifford R, Edelmann J, Blakemore S, et al. Genomic Disruption of the Histone Methyltransferase SETD2 in Chronic Lymphocytic Leukaemia. Leukemia (2016) 30:2179-86. doi: 10.1038/leu.2016.134

21. Cortés-Ciriano I, Lee JJ-K, Xi R, Jain D, Jung YL, Yang L, et al. Comprehensive Analysis of Chromothripsis in 2,658 Human Cancers Using Whole-Genome Sequencing. Nat Genet (2020) 52:331-41. doi: 10.1038/s41588-019-0576-7

22. Crasta K, Ganem NJ, Dagher R, Lantermann AB, Ivanova EV, Pan Y, et al. DNA Breaks and Chromosome Pulverization From Errors in Mitosis. Nature (2012) 482:53-8. doi: 10.1038/nature10802 
23. Maciejowski J, Li Y, Bosco N, Campbell PJ, de Lange T. Chromothripsis and Kataegis Induced by Telomere Crisis. Cell (2015) 163:1641-54. doi: 10.1016/ j.cell.2015.11.054

24. Ramos-Campoy S, Puiggros A, Beà S, Bougeon S, Larráyoz MJ, Costa D, et al. Chromosome Banding Analysis and Genomic Microarrays Are Both Useful But Not Equivalent Methods for Genomic Complexity Risk Stratification in Chronic Lymphocytic Leukemia Patients. Haematologica (2020). doi: 10.3324/haematol.2020.274456

25. Hynst J, Plevova K, Radova L, Bystry V, Pal K, Pospisilova S. Bioinformatic Pipelines for Whole Transcriptome Sequencing Data Exploitation in Leukemia Patients With Complex Structural Variants. PeerJ (2019) 7:e7071. doi: $10.7717 /$ peerj.7071

26. Kim T-M, Xi R, Luquette LJ, Park RW, Johnson MD, Park PJ. Functional Genomic Analysis of Chromosomal Aberrations in a Compendium of 8000 Cancer Genomes. Genome Res (2013) 23:217-27. doi: 10.1101/gr.140301.112

27. Stevens-Kroef M, Weghuis DO, Croockewit S, Derksen L, Hooijer J, ElIdrissiZaynoun N, et al. High Detection Rate of Clinically Relevant Genomic Abnormalities in Plasma Cells Enriched From Patients With Multiple Myeloma. Genes Chromosomes Cancer (2012) 51:997-1006. doi: 10.1002/ gcc. 21982

28. Zemanova Z, Michalova K, Buryova H, Brezinova J, Kostylkova K, Bystricka D, et al. Involvement of Deleted Chromosome 5 in Complex Chromosomal Aberrations in Newly Diagnosed Myelodysplastic Syndromes (MDS) Is Correlated With Extremely Adverse Prognosis. Leukemia Res (2014) 38:537-44. doi: 10.1016/j.leukres.2014.01.012

29. Abáigar M, Robledo C, Benito R, Ramos F, Díez-Campelo M, Hermosín L, et al. Chromothripsis Is a Recurrent Genomic Abnormality in High-Risk Myelodysplastic Syndromes. PloS One (2016) 11:e0164370. doi: 10.1371/ journal.pone. 0164370

30. Bochtler T, Granzow M, Stölzel F, Kunz C, Mohr B, Kartal-Kaess M, et al. Marker Chromosomes can Arise From Chromothripsis and Predict Adverse Prognosis in Acute Myeloid Leukemia. Blood (2017) 129:1333-42. doi: 10.1182/blood-2016-09-738161

31. Rücker FG, Dolnik A, Blätte TJ, Teleanu V, Ernst A, Thol F, et al. Chromothripsis is Linked to TP53 Alteration, Cell Cycle Impairment, and Dismal Outcome in Acute Myeloid Leukemia With Complex Karyotype. Haematologica (2018) 103:e17-20. doi: 10.3324/haematol.2017.180497

32. Fontana MC, Marconi G, Feenstra JDM, Fonzi E, Papayannidis C, Ghelli Luserna di Rorá A, et al. Chromothripsis in Acute Myeloid Leukemia: Biological Features and Impact on Survival. Leukemia (2018) 32:1609-20. doi: 10.1038/s41375-018-0035-y

33. MacKinnon RN, Campbell LJ. Chromothripsis Under the Microscope: A Cytogenetic Perspective of Two Cases of AML With Catastrophic Chromosome Rearrangement. Cancer Genet (2013) 206:238-51. doi: 10.1016/j.cancergen.2013.05.021

34. Forero-Castro M, Robledo C, Benito R, Abáigar M, África Martín A, Arefi M, et al. Genome-Wide DNA Copy Number Analysis of Acute Lymphoblastic Leukemia Identifies New Genetic Markers Associated With Clinical Outcome. PloS One (2016) 11:e0148972. doi: 10.1371/journal.pone.0148972

35. Zhang J, Ding L, Holmfeldt L, Wu G, Heatley SL, Payne-Turner D, et al. The Genetic Basis of Early T-Cell Precursor Acute Lymphoblastic Leukaemia. Nature (2012) 481:157-63. doi: 10.1038/nature10725

36. Li Y, Schwab C, Ryan S, Papaemmanuil E, Robinson HM, Jacobs P, et al. Constitutional and Somatic Rearrangement of Chromosome 21 in Acute Lymphoblastic Leukaemia. Nature (2014) 508:98-102. doi: 10.1038/ nature 13115

37. Ratnaparkhe M, Hlevnjak M, Kolb T, Jauch A, Maass KK, Devens F, et al. Genomic Profiling of Acute Lymphoblastic Leukemia in Ataxia Telangiectasia Patients Reveals Tight Link Between ATM Mutations and Chromothripsis. Leukemia (2017) 31:2048-56. doi: 10.1038/leu.2017.55

38. Puente XS, Beà S, Valdés-Mas R, Villamor N, Gutiérrez-Abril J, MartínSubero JI, et al. Non-Coding Recurrent Mutations in Chronic Lymphocytic Leukaemia. Nature (2015) 526:519-24. doi: 10.1038/nature14666

39. Burns A, Alsolami R, Becq J, Stamatopoulos B, Timbs A, Bruce D, et al. Whole-Genome Sequencing of Chronic Lymphocytic Leukaemia Reveals Distinct Differences in the Mutational Landscape Between IgHVmut and IgHVunmut Subgroups. Leukemia (2018) 32:332-42. doi: 10.1038/ leu.2017.177
40. Chudasama P, Mughal SS, Sanders MA, Hübschmann D, Chung I, Deeg KI, et al. Integrative Genomic and Transcriptomic Analysis of Leiomyosarcoma. Nat Commun (2018) 9:144. doi: 10.1038/s41467-017-02602-0

41. ICGC/TCGA Pan-Cancer Analysis of Whole Genomes Consortium. PanCancer Analysis of Whole Genomes. Nature (2020) 578:82-93. doi: 10.1038/ s41586-020-1969-6

42. Barrett T, Troup DB, Wilhite SE, Ledoux P, Rudnev D, Evangelista C, et al. NCBI GEO: Archive for High-Throughput Functional Genomic Data. Nucleic Acids Res (2009) 37:D885-890. doi: 10.1093/nar/gkn764

43. Voronina N, Wong JKL, Hübschmann D, Hlevnjak M, Uhrig S, Heilig CE, et al. The Landscape of Chromothripsis Across Adult Cancer Types. Nat Commun (2020) 11:2320. doi: 10.1038/s41467-020-16134-7

44. Horak P, Klink B, Heining C, Gröschel S, Hutter B, Fröhlich M, et al. Precision Oncology Based on Omics Data: The NCT Heidelberg Experience. Int $J$ Cancer (2017) 141:877-86. doi: 10.1002/ijc.30828

45. Li Y, Buijs-Gladdines JGCAM, Canté-Barrett K, Stubbs AP, Vroegindeweij EM, Smits WK, et al. IL-7 Receptor Mutations and Steroid Resistance in Pediatric T Cell Acute Lymphoblastic Leukemia: A Genome Sequencing Study. PloS Med (2016) 13:e1002200. doi: 10.1371/journal.pmed.1002200

46. Döhner H, Stilgenbauer S, Benner A, Leupolt E, Kröber A, Bullinger L, et al. Genomic Aberrations and Survival in Chronic Lymphocytic Leukemia. N Engl J Med (2000) 343:1910-6. doi: 10.1056/NEJM200012283432602

47. Stilgenbauer S, Schnaiter A, Paschka P, Zenz T, Rossi M, Döhner K, et al. Gene Mutations and Treatment Outcome in Chronic Lymphocytic Leukemia: Results From the CLL8 Trial. Blood (2014) 123:3247-54. doi: 10.1182/ blood-2014-01-546150

48. Hallek M, Cheson BD, Catovsky D, Caligaris-Cappio F, Dighiero G, Döhner $\mathrm{H}$, et al. Guidelines for the Diagnosis and Treatment of Chronic Lymphocytic Leukemia: A Report From the International Workshop on Chronic Lymphocytic Leukemia Updating the National Cancer Institute-Working Group 1996 Guidelines. Blood (2008) 111:5446-56. doi: 10.1182/blood-200706-093906

49. Rossi D, Khiabanian H, Spina V, Ciardullo $\mathrm{C}$, Bruscaggin $\mathrm{A}$, Famà R, et al. Clinical Impact of Small TP53 Mutated Subclones in Chronic Lymphocytic Leukemia. Blood (2014) 123:2139-47. doi: 10.1182/blood-2013-11-539726

50. International CLL-IPI Working Group. An International Prognostic Index for Patients With Chronic Lymphocytic Leukaemia (CLL-IPI): A Meta-Analysis of Individual Patient Data. Lancet Oncol (2016) 17:779-90. doi: 10.1016/ S1470-2045(16)30029-8

51. Malcikova J, Stano-Kozubik K, Tichy B, Kantorova B, Pavlova S, Tom N, et al. Detailed Analysis of Therapy-Driven Clonal Evolution of TP53 Mutations in Chronic Lymphocytic Leukemia. Leukemia (2015) 29:877-85. doi: 10.1038/ leu.2014.297

52. Ma X, Liu Y, Liu Y, Alexandrov LB, Edmonson MN, Gawad C, et al. PanCancer Genome and Transcriptome Analyses of 1,699 Paediatric Leukaemias and Solid Tumours. Nature (2018) 555:371-6. doi: 10.1038/nature25795

53. Forbes SA, Bindal N, Bamford S, Cole C, Kok CY, Beare D, et al. COSMIC: Mining Complete Cancer Genomes in the Catalogue of Somatic Mutations in Cancer. Nucleic Acids Res (2011) 39:D945-50. doi: 10.1093/nar/gkq929

54. Vogelstein B, Lane D, Levine AJ. Surfing the P53 Network. Nature (2000) 408:307-10. doi: 10.1038/35042675

55. Finn WG, Kay NE, Kroft SH, Church S, Peterson LC. Secondary Abnormalities of Chromosome 6q in B-Cell Chronic Lymphocytic Leukemia: A Sequential Study of Karyotypic Instability in 51 Patients. Am J Hematol (1998) 59:223-9. doi: 10.1002/(sici)1096-8652(199811)59:3<223:: aid-ajh7>3.0.co;2-y

56. Forconi F, Rinaldi A, Kwee I, Sozzi E, Raspadori D, Rancoita PMV, et al. Genome-Wide DNA Analysis Identifies Recurrent Imbalances Predicting Outcome in Chronic Lymphocytic Leukaemia With $17 \mathrm{p}$ Deletion. $\mathrm{Br} J$ Haematol (2008) 143:532-6. doi: 10.1111/j.1365-2141.2008.07373.x

57. Brown JR, Hanna M, Tesar B, Werner L, Pochet N, Asara JM, et al. Integrative Genomic Analysis Implicates Gain of PIK3CA at 3q26 and MYC at 8q24 in Chronic Lymphocytic Leukemia. Clin Cancer Res (2012) 18:3791-802. doi: 10.1158/1078-0432.CCR-11-2342

58. Kume K, Iizumi Y, Shimada M, Ito Y, Kishi T, Yamaguchi Y, et al. Role of NEnd Rule Ubiquitin Ligases UBR1 and UBR2 in Regulating the leucine-mTOR Signaling Pathway. Genes Cells (2010) 15:339-49. doi: 10.1111/j.13652443.2010.01385.x 
59. Jebaraj BMC, Stilgenbauer S. Telomere Dysfunction in Chronic Lymphocytic Leukemia. Front Oncol (2021) 0:612665. doi: 10.3389/fonc.2020.612665

60. Olbertova H, Plevova K, Stranska K, Pospisilova S. Telomere Dynamics in Adult Hematological Malignancies. BioMed Pap Med Fac Univ Palacky Olomouc Czech Repub (2019) 163:1-7. doi: 10.5507/bp.2018.084

61. Sharpless NE, Sherr CJ. Forging a Signature of In Vivo Senescence. Nat Rev Cancer (2015) 15:397-408. doi: 10.1038/nrc3960

62. Falandry C, Bonnefoy M, Freyer G, Gilson E. Biology of Cancer and Aging: A Complex Association With Cellular Senescence. J Clin Oncol (2014) 32:260410. doi: 10.1200/JCO.2014.55.1432

63. Damle RN, Batliwalla FM, Ghiotto F, Valetto A, Albesiano E, Sison C, et al. Telomere Length and Telomerase Activity Delineate Distinctive Replicative Features of the B-CLL Subgroups Defined by Immunoglobulin V Gene Mutations. Blood (2004) 103:375-82. doi: 10.1182/blood-2003-04-1345

64. Roos G, Kröber A, Grabowski P, Kienle D, Bühler A, Döhner H, et al. Short Telomeres Are Associated With Genetic Complexity, High-Risk Genomic Aberrations, and Short Survival in Chronic Lymphocytic Leukemia. Blood (2008) 111:2246-52. doi: 10.1182/blood-2007-05-092759

65. Rossi D, Lobetti Bodoni C, Genuardi E, Monitillo L, Drandi D, Cerri M, et al. Telomere Length Is an Independent Predictor of Survival, Treatment Requirement and Richter's Syndrome Transformation in Chronic Lymphocytic Leukemia. Leukemia (2009) 23:1062-72. doi: 10.1038/leu.2008.399

66. Bechter OE, Eisterer W, Pall G, Hilbe W, Kühr T, Thaler J. Telomere Length and Telomerase Activity Predict Survival in Patients With B Cell Chronic Lymphocytic Leukemia. Cancer Res (1998) 58:4918-22.

67. Damle RN, Temburni S, Banapour T, Paul S, Mongini PKA, Allen SL, et al. Chiorazzi N. T-Cell Independent, B-Cell Receptor-Mediated Induction of Telomerase Activity Differs Among IGHV Mutation-Based Subgroups of Chronic Lymphocytic Leukemia Patients. Blood (2012) 120:2438-49. doi: 10.1182/blood-2012-02-409110

68. Lin TT, Letsolo BT, Jones RE, Rowson J, Pratt G, Hewamana S, et al. Telomere Dysfunction and Fusion During the Progression of Chronic Lymphocytic Leukemia: Evidence for a Telomere Crisis. Blood (2010) 116:1899-907. doi: 10.1182/blood-2010-02-272104

69. Lo AWI, Sabatier L, Fouladi B, Pottier G, Ricoul M, Murnane JP. DNA Amplification by Breakage/Fusion/Bridge Cycles Initiated by Spontaneous Telomere Loss in a Human Cancer Cell Line. Neoplasia (2002) 4:531-8. doi: 10.1038/sj.neo.7900267

70. Ernst A, Jones DTW, Maass KK, Rode A, Deeg KI, Jebaraj BMC, et al. Telomere Dysfunction and Chromothripsis. Int J Cancer (2016) 138:2905-14. doi: $10.1002 / \mathrm{ijc} .30033$

71. Dos Santos P, Panero J, Palau Nagore V, Stanganelli C, Bezares RF, Slavutsky I. Telomere Shortening Associated With Increased Genomic Complexity in Chronic Lymphocytic Leukemia. Tumour Biol (2015) 36:8317-24. doi: 10.1007/s13277-015-3556-2

72. Thomay K, Fedder C, Hofmann W, Kreipe H, Stadler M, Titgemeyer J, et al. Telomere Shortening, TP53 Mutations and Deletions in Chronic Lymphocytic Leukemia Result in Increased Chromosomal Instability and Breakpoint Clustering in Heterochromatic Regions. Ann Hematol (2017) 96:1493-500. doi: 10.1007/s00277-017-3055-1

73. Jebaraj BMC, Tausch E, Landau DA, Bahlo J, Robrecht S, Taylor-Weiner AN, et al. Short Telomeres Are Associated With Inferior Outcome, Genomic Complexity, and Clonal Evolution in Chronic Lymphocytic Leukemia. Leukemia (2019) 33:2183-94. doi: 10.1038/s41375-019-0446-4

74. Mansouri L, Grabowski P, Degerman S, Svenson U, Gunnarsson R, Cahill N, et al. Short Telomere Length Is Associated With NOTCH1/SF3B1/TP53 Aberrations and Poor Outcome in Newly Diagnosed Chronic Lymphocytic Leukemia Patients. Am J Hematol (2013) 88:647-51. doi: 10.1002/ajh.23466
75. Norris K, Hillmen P, Rawstron A, Hills R, Baird DM, Fegan CD, et al. Telomere Length Predicts for Outcome to FCR Chemotherapy in CLL. Leukemia (2019) 33:1953-63. doi: 10.1038/s41375-019-0389-9

76. Strefford JC, Kadalayil L, Forster J, Rose-Zerilli MJJ, Parker A, Lin TT, et al. Telomere Length Predicts Progression and Overall Survival in Chronic Lymphocytic Leukemia: Data From the UK LRF CLL4 Trial. Leukemia (2015) 29:2411-4. doi: 10.1038/leu.2015.217

77. Song DY, Kim J-A, Jeong D, Yun J, Kim S-M, Lim K, et al. Telomere Length and Its Correlation With Gene Mutations in Chronic Lymphocytic Leukemia in a Korean Population. PloS One (2019) 14:e0220177. doi: 10.1371/ journal.pone. 0220177

78. Britt-Compton B, Lin TT, Ahmed G, Weston V, Jones RE, Fegan C, et al. Extreme Telomere Erosion in ATM-Mutated and 11q-Deleted CLL Patients is Independent of Disease Stage. Leukemia (2012) 26:826-30. doi: 10.1038/ leu.2011.281

79. Steinbrecher D, Jebaraj BMC, Schneider C, Edelmann J, Cymbalista F, Leblond V, et al. Telomere Length in Poor-Risk Chronic Lymphocytic Leukemia: Associations With Disease Characteristics and Outcome. Leuk Lymphoma (2018) 59:1614-23. doi: 10.1080/10428194.2017.1390236

80. Ivkov R, Bunz F. Pathways to Chromothripsis. Cell Cycle (2015) 14:2886-90. doi: $10.1080 / 15384101.2015 .1068483$

81. Le DT, Uram JN, Wang H, Bartlett BR, Kemberling H, Eyring AD, et al. PD-1 Blockade in Tumors With Mismatch-Repair Deficiency. N Engl J Med (2015) 372:2509-20. doi: 10.1056/NEJMoa1500596

82. Mansfield AS, Peikert T, Smadbeck JB, Udell JBM, Garcia-Rivera E, Elsbernd L, et al. Neoantigenic Potential of Complex Chromosomal Rearrangements in Mesothelioma. J Thorac Oncol (2019) 14:276-87. doi: 10.1016/ j.jtho.2018.10.001

83. Singh ZN, Richards S, El Chaer F, Duong VH, Gudipati MA, Waters EO, et al. Cryptic ETV6-PDGFRB Fusion in a Highly Complex Rearrangement of Chromosomes 1, 5, and 12 Due to a Chromothripsis-Like Event in a Myelodysplastic Syndrome/Myeloproliferative Neoplasm. Leukemia Lymphoma (2019) 60:1304-7. doi: 10.1080/10428194.2018.1480774

84. Arber DA, Orazi A, Hasseriian R, Thiele J, Borowitz MJ, Le Beau MM, et al. The 2016 Revision to the World Health Organization Classification of Myeloid Neoplasms and Acute Leukemia. Blood (2016) 127:2391-405. doi: 10.1182/blood-2016-03-643544

85. O'Neil NJ, Bailey ML, Hieter P. Synthetic Lethality and Cancer. Nat Rev Genet (2017) 18:613-23. doi: 10.1038/nrg.2017.47

86. Lord CJ, Ashworth A. The DNA Damage Response and Cancer Therapy. Nature (2012) 481:287-94. doi: 10.1038/nature10760

Conflict of Interest: The authors declare that the research was conducted in the absence of any commercial or financial relationships that could be construed as a potential conflict of interest.

Publisher's Note: All claims expressed in this article are solely those of the authors and do not necessarily represent those of their affiliated organizations, or those of the publisher, the editors and the reviewers. Any product that may be evaluated in this article, or claim that may be made by its manufacturer, is not guaranteed or endorsed by the publisher.

Copyright $\odot 2021$ Zavacka and Plevova. This is an open-access article distributed under the terms of the Creative Commons Attribution License (CC BY). The use, distribution or reproduction in other forums is permitted, provided the original author(s) and the copyright owner(s) are credited and that the original publication in this journal is cited, in accordance with accepted academic practice. No use, distribution or reproduction is permitted which does not comply with these terms. 